\title{
Models for forecasting hospital bed requirements in the acute sector
}

\author{
R D T Farmer, J Emami
}

\begin{abstract}
Study objective-The aim was to evaluate the current approach to forecasting hospital bed requirements.

Design-The study was a time series and regression analysis. The time series for mean duration of stay for general surgery in the age group 15-44 years (1969-1982) was used in the evaluation of different methods of forecasting future values of mean duration of stay and its subsequent use in the formation of hospital bed requirements.

Results-It has been suggested that the simple trend fitting approach suffers from model specification error and imposes unjustified restrictions on the data. Time series approach (Box-Jenkins method) was shown to be a more appropriate way of modelling the data.

Conclusion-The simple trend fitting approach is inferior to the time series approach in modelling hospital bed requirements.
\end{abstract}

Over the past 25 years there have been substantial changes in medical practice which have altered gross demand for hospital facilities, the type of facilities required, and the ways they are used. All these changes have financial implications both in terms of revenue requirements and capital investment. During the same period there has been a move to ensure that health care facilities are more evenly distributed within the country as a whole. Planning problems are compounded by the fact that many hospitals are old and reaching the end of their useful life. Thus planning of hospital services is an important issue, yet the models available to forecast facilities and manpower are generally inadequate. There is a need to develop a method whereby the optimum level of provision can be established and the likely effects of changes proposed can be assessed. A planning model should enable managers to maximise the use of projected capital and investment and minimise cost.

This paper is concerned with one aspect of the development of such models, namely, obtaining short term forecasts of future work loads in the acute hospital sector. It does not evaluate current methods for forecasting populations.

The plan of this paper is as follows: the first section considers the relationship used to forecast hospital bed needs and the data used for this purpose; the second section introduces the methodology of forecasting used throughout the paper; the third section is devoted to a critical analysis of the current method of forecasting hospital bed requirements and illustrates the time series approach by applying it to a specific time series of Mean Duration of Stay (MDS); the fourth section considers the joint estimation of the ingredients of the relationship used for bed forecasting, Mean Duration of Stay, and Admission Rate (AR) as part of multiequation model; the conclusion is presented in the final section.

\section{Data}

Hospital bed requirements are usually forecast with the aid of the following relationship:

$$
\text { Beds }=(P o p \star A R \star M D S \star E) / 365
$$

where: Beds = forecast number of available beds; $\mathrm{AR}=$ projected admission rate (the forecast discharge rate is usually used as a proxy); MDS $=$ projected average duration of hospital stay; Pop = projected population to be served; and $E=$ projected efficiency factor. This may be the percentage daily occupancy or the so called turnover interval.

The daily available beds are defined as those beds available for the treatment of patients. The bed complement is a higher number that takes account of the fact that additional beds will be required because, for example, there will be a need to refurbish and update hospital facilities and slack to allow for emergencies or disasters. Such changes will result in some of the beds being unavailable most of the time. Sometimes an adjustment for the difference between the required available beds and the required bed complement is incorporated in the basic model.

The simple identity (equation 1) is usually applied to different age, sex, and specialty groups. This is essential as the process determining the ingredients of the identity changes between these groups. However, if disaggregation of the data is taken too far then the potential gain is lost due to lack of data.

The efficiency factors used in forecasts are essentially (but not necessarily) arbitrary. Since MDS and AR are critical to the whole model it is essential to understand how they are derived.

\section{MEAN DURATION OF STAY}

The mean duration of stay can be calculated in one of two ways. It can either be generated from data collected on discharge of patients from hospital (Hospital Activity Analysis data), referred to as patient orientated data, or estimated from statistics on hospital bed utilisation ( $\mathrm{SH} 3$ data)bed orientated data. 
Average lengths of stay are calculated from patient orientated data as follows. A form is completed in respect of all patients admitted to and discharged from acute (and most other) hospitals administered by the NHS. The admission and discharge forms include personal identification data to allow them to be linked. From these forms it is possible to establish the number of days spent in hospital by individual patients during each episode of admission. The mean duration of stay is the sum of days spent in hospital by patients discharged during that period. That is:

$$
\operatorname{MDS}=\Sigma(\operatorname{LOS}) / P
$$

Where: $L O S=$ total number of days spent in hospital by each individual patient; and $\mathrm{P}=$ the total number of patients.

The distribution of patients by exact length of stay over time within most specialties and age and sex groups is unimodal and skewed to the right. In most age/sex/specialty groups the mode is around two to three days (figs 1 and 2). Thus changes over time in the arithmetical mean length of stay tend to be caused by changes in the thickness of the tail rather than the location of the mode. It may be deduced that the mean is likely to be a relatively poor indicator of the change. In view of the limitations imposed by the distribution of lengths of stay it is unlikely that the identity (equation 1) can be used with any degree of accuracy for forecasting the numbers of beds that may be acquired in some future year. However, despite this reservation, it is possible to improve on the current methods used in forecasting mean duration of stay.

Bed orientated data are different and less revealing of the underlying process. Here the

Figure 1 Distribution of patients by length of stay and age (15-44 years): general surgery

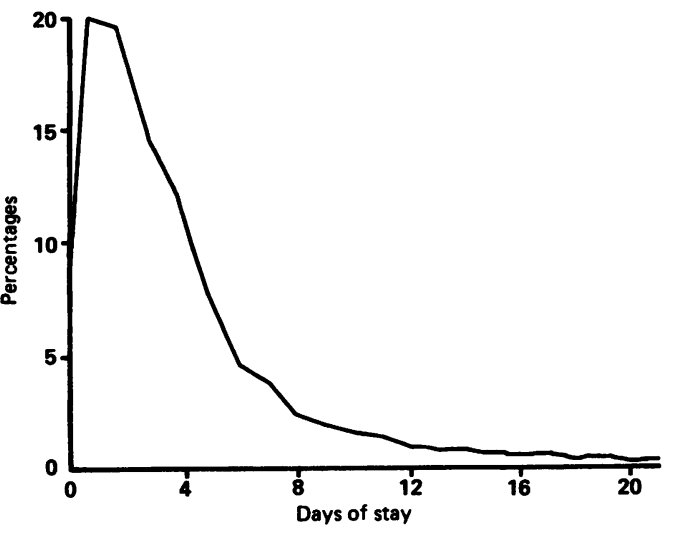

Figure 2 Distribution of patients by length of stay and age (15-44 years, log percentages): : general surgery mean duration of stay is estimated by dividing the product of the average number of beds occupied daily during a period and the number of days in that period by the number of discharges (and deaths) that occurred. This method uses SH3 type data. It tells us nothing about the distribution of patients by length of stay. A further major problem is that data on bed occupancy by age and sex of patients and precise specialty in which they were treated are not normally available from this source. There are important differences in the way certain manoeuvres within the hospital are handled by the two information systems. In Hospital Activity Analysis data, transfer of a patient from one doctor's care to another's is usually recorded as a discharge and immediate readmission; the same is not true of $\mathrm{SH} 3$ data.

\section{ADMISSION RATE}

The hospital admission rate is not normally presented in routine reports. Data are presented as discharges (and deaths), and discharge (and death) rates. These are used as proxies for admissions and admission rates. The potential for error by using these proxies in the "acute" specialties is small. Few patients stay in hospital for over a year; and year to year changes in admissions are not great. Rates are calculated by dividing the number of admissions by the catchment population of the hospital, district, region, or the country as a whole. There is no real denominator problem with national data. With subnational population groupings, the definition of the denominator can present difficulties as the population "at risk" to be admitted is hard to define. There are frequent overlapping catchment areas; the effective catchment population for one specialty/age/sex group may differ from that for another and confusion may arise regarding the use of the actual population or the theoretical (or desirable) population base.

COMMENTS ON DATA USED IN THE PRESENT METHOD At present national data are used to forecast the parameters (mean duration of stay and admission rate) which are then applied at regional or district levels. The implicit assumption here is that, in any given year, the gross national provision of hospital beds is optimal, although no assumption is made regarding the distribution of this nationally "optimal" provision within the country. Thus the forecasts produced for a particular district (or region) give an indication of what resources will be required at some future time, assuming the factors that determine the national optimum provision will continue to be effective during the period of the forecast.

Moreover, it is assumed that the optimum admission rate and mean duration of stay are uniform throughout the country. Underlying this is an assumption that net morbidity is identical and that the treatment requirements (in terms of length of stay and numbers of admissions) do not vary from place to place. But there is much evidence that patterns of morbidity vary between the NE and SE of England ${ }^{45}$ and within regions and that patients from different social and occupational backgrounds vary in both the number of days they require in hospital for an episode of illness and the numbers of admissions 
that may be required per episode. ${ }^{6}$ The problems of weighting forecasts, or even current provision, for variations in social circumstances and morbidity ${ }^{7}$ have been addressed by many investigators but to date no satisfactory method has been devised.

\section{Forecasting methodology}

It is instructive to have a general overview of the two methods of forecasting referred to in this paper, before discussing them in the context of forecasting of mean duration of stay and admission rates, and their use in determination of number of beds. The two methods are: (1) forecasting from a structural model; and (2) the time series or Box-Jenkins ${ }^{1}$ method.

FORECASTING FROM A STRUCTURAL MODEL

Suppose we are in year $n$, and we require the forecast of a quantity $y$, for year $n+l$. The first step in this method is to construct a model by relating $y$ to one or more variables $x$, for which future values are more readily available. Let us denote the relationship by a function $f$. So that the model can be written as:

$$
y_{t}=f\left(x_{t}\right)+\varepsilon_{t}
$$

In this equation, $\varepsilon$ is an unobservable disturbance term, indicating that we do not expect data to satisfy $f$ exactly, and the subscript $t$ refers to the value of the variables and disturbances at time $t$. Note that $x_{t}$ can be a vector of variables and can include past values of $y$ (ie, lags denoted by $y_{t-k}$, with $k>=1$ ), as well as a trend $t$. The next step in this procedure is to specify the relationship $f$ in terms of $x_{t}$ and some estimable parameters. Many mathematical functions can be used, for example $f$ can be specified as a polynomial in $x$ or as an exponential function of $x$. Finally using our information (data) on $x, y$ up to present time $n$, we estimate parameters determining $f$, using some appropriate estimation technique (eg, linear or nonlinear regression). To calculate the future value of $y$ at year $n+l$, given $x_{n+l}$, we.substitute $x_{n+l}$, into the right hand side of equation (2), set $\varepsilon_{n+l}$, to zero (its expected value) and read the required value of $y$, (ie, $y_{n+l}$ ). Note that the distribution of the disturbance term $\varepsilon$ in equation (2) enables us to put probability bounds for the estimated parameters of $f$, as well as our forecast $y_{n+l}$. This is exactly what is currently being used in forecasting mean duration of stay and admission rate, with $x_{t}=t$ and $f$ exponential (see below, "Present methods of forecasting"). It is also instructive to bear in mind that equation (2) can be regarded as one equation of a system of equations determining $y$, in which case we may have to use more sophisticated estimation procedures such as two stage least squares, or two stage non-linear least squares, depending on the specification of $f$.

This method has many advantages, the most notable of which is that it implies a cause and effect relationship which can be explored further. However from a forecasting point of view it has three major defects: (1) To obtain a forecast of $y$ we need future value of $x$, so as Nelson ${ }^{2}$ puts it, "It would appear that the structural model has transformed rather than solved the forecasting problem, that is, it transformed it into the problem of forecasting (exogeneous variables:x)". (2) The distribution of the disturbance term $\varepsilon$ in equation (2) may be wrongly specified, which in turn induces error in estimated parameters of $f$ as well as forecasted values of $y$. Note that misspecification of the distribution of the disturbance term in equation (2) is equivalent to misspecifying distribution of $y$ (see below, "Present methods of forecasting", on this point in relation to mean duration of stay). ${ }^{3}$ The relationship $f$ may be inadequate and impose unnecessary restriction on the data (see also below).

TIME SERIES OR BOX-JENKINS METHOD OF FORECASTING

It is important to realise that a time series of length $\boldsymbol{n}$ is not $\boldsymbol{n}$ different observations of a random variable, but one observation of a multivariate random variable. This dependence over time is a mixed blessing. On the one hand, standard methods requiring, independently, identically distributed observations cannot be used directly; on the other hand the dependence over time can be exploited for several purposes, not the least of which is forecasting.

Following our previous notation, in time series analysis we are looking for the specification of the joint probability distribution of the sequence of observations $y_{1}, y_{2}, \ldots, y_{n}$ : let us denote this distribution by $P_{1} \ldots n\left(y, y_{2}, y_{n}\right)$ and note that in general the parameter, or even the form of this density, may depend on the time point in question. This means that a sequence of observations evolves through time, according to a probability law $P$. Knowledge of $P$ will enable us to make inference about future likely values of $y$. The Box-Jenkins method is a procedure for identifying $P$. This is done in three stages:

(1) Identification-At this stage we entertain a set of possible models for the generating process of $y$, by comparison of the sample correlogram, ie, plot of covariance of $y_{t}$, and $y_{t-k}(k>0)$ against $k$ for a few lags $(k)$; with the correlogram of some known theoretical models.

(2) Estimation-In this stage we estimate the models that are entertained in stage (1). The basic idea is to determine the parameter values that minimise the sum of the squared residuals:

$$
\sum_{t=1}^{n} \varepsilon_{t}{ }^{2}
$$

Where $\varepsilon_{t}$, is the residual of the model entertained. For example for the ARMA (equation 1) model of equation (2) below we have:

$$
\varepsilon_{t}=y_{t}-\alpha y_{t-1}-\Theta \varepsilon_{t-1}
$$

This usually requires a non-linear optimisation routine. The algorithm of Marquardt is among the most popular ones. To start the iterative procedure an initial estimate of the parameters $(\alpha, \Theta)$ as well as values for presample period $y_{o}$ and $\varepsilon_{o}$ are needed. There are many ways to assign these values. One simple way is to set $y_{o}$ and $\varepsilon_{o}$ to zero and compute the initial values for parameters by equating the sample correlogram at different 
lags to their theoretical counterpart and solving the resulting equations. In practice Managerial and Statistical packages such as SAS, statgraphics, etc, take care of these problems and are easily accessible (see ${ }^{12}$ for more details on these issues; ${ }^{2}$ is especially relevant to managers). (3) Diagnostic checking-This involves applying tests to see if the model estimated in stage (2) fits the data well. One way to do this is to form the correlogram of the estimated residuals $e_{t}$ :

$$
r_{k}=\frac{\sum_{t} e_{t} e_{t-k}}{\sum e_{t}^{2}}
$$

and compute the Box-Pierce statistics $Q=\left(r_{1}{ }^{2}+r_{2}{ }^{2}+\ldots+r_{m}{ }^{2}\right) x T$ ( $T$ is the number of observations) for say $m=8$ to 10 for small models. Then for an $\operatorname{ARMA}(p, q)$ model, $Q \sim X^{2}(m-p-q)$. Large values of $Q$ reject the null hypothesis of no serial correlation (see ${ }^{12}$ for more detail). Alternatively we may save few observation points in the estimation stage and see how the estimated model predicts these values.

In the above discussion we assumed that the series under investigation is stationary, ie, the extent to which two terms in the series are related depends only on the time interval between them and not on the time itself. Few series in practice satisfy this condition, especially if they are levels rather than rates of change. To overcome this difficulty we difference the series first before applying the above procedures. (For an excellent non-technical discussion of the Box-Jenkins approach for managerial purposes see Granger $^{3}$.) Now suppose that the above procedure suggests the following simple model:

$$
y_{t}=\alpha y_{t-1}+\varepsilon_{t}+\Theta \varepsilon_{t-1}
$$

This equation is known as a first order autoregressive moving average model. It is autoregressive because it involves the term $y_{t-1}$, and moving average because $\left(\varepsilon_{t}+\Theta \varepsilon_{t-1}\right)$ is a moving average. We first form the forecast of $y_{n+1}$ (one step ahead forecast of $y$ ), substitute $n+1$ for $t$ in equation ( 3 ) to get:

$$
y_{n+1}=\alpha y_{n}+\varepsilon_{n+1}+\Theta \varepsilon_{n}
$$

Since we do not know $\varepsilon_{n+1}$ we set it to its expected value zero, and $\varepsilon_{n}$ to the estimated residual for time $n$. To obtain the forecast of $y_{n+l}(l>1)$, repeat the procedure by putting $n+l$ for $t$ in equation (2) to obtain the recursive formula:

$$
y_{n+l}=\alpha y_{n+l-1}
$$

and use the value calculated from equation (4) as the starting value. All this is readily available in most managerial packages. It is the model we identified below for mean duration of stay.

In conclusion it is important to realise that much of the information content of data is used in equation (3) to form future forecasts than in a simple deterministic trend in $t$ model. This is because a deterministic trend such as:

$$
y_{t}=\alpha+\beta t
$$

implies that:

$$
y_{t}-y_{t-1}=\beta \text {, a constant }
$$

If we rewrite equation (4) as:

$$
y_{0}-\alpha y_{t-1}=\varepsilon_{t}+\Theta \varepsilon_{t-1}
$$

and compare it with equation (7) we see that equation (8) uses the data to estimate $\alpha$ and $\Theta$, while (7) sets $\alpha=1$ a priori, and makes no attempt to model the residuals, ie, (7) says that the time series is deterministic. The same argument also applies to any polynomial trend.

Present methods of forecasting mean duration of stay and admission rate In this section we discuss the limitation of the currently applied method of forecasting mean duration of stay and admission rate. We suggest that these limitations are so substantial as to seriously limit their use in the managerial decision making processes, and we apply the Box-Jenkins method to a series of mean duration of stay for general surgery.

Within the simple identity (equation 1) the mean duration of stay and admission rate are projected separately. The limitations imposed then are similar; throughout we use mean duration of stay to illustrate our points.

It is usual to arrive at projected values for mean duration of stay by first fitting historical observation to a log-linear curve using the method of ordinary least squares, ie, estimating the coefficient $a$ and $b$ of the following equation:

$$
\log (M D S)=a+b t
$$

In so doing $a$ and $b$ are considered not to depend on time and $t$ is time.

The fitted curve is then extrapolated to give a "value" for the desired year; this is then used in equation (1) to arrive at the forecast of beds required.

Recall our discussion of single equation structural forecasting; there we pointed out that misspecification of the distribution of the disturbance term $\varepsilon$ in equation (2) is equivalent to misspecification of the distribution of the dependent variable $y$, ie, mean duration of stay in our context here. We have already pointed out that the distribution of the dependent variable is skewed. This is not so important with admission rate since admissions are observed numbers and the variance accrues from the denominator (the population). The size of the population is normally so large that the variance of the rate would be small. Skewing is much more important with mean duration of stay. Here the problem arises from the distribution from which the value is derived.

Log normal distribution for the error term may not be the most suitable distribution as implied by equation (9), for the following reason: equation (9) can be rewritten as

$$
y=a(1-g)^{t}
$$


where $a$ and $g$ are constants, and $y=$ mean duration of stay, where $0<g<1$ and $a>0$. If $g>1$ then $(1-g)<0$, which would contradict the fact that $y$ must be greater than 0 . On the other hand if $g<0$ then $(1-g)>1$ and the process would explode as time passes.

The crucial point to note about equation $(10)$ is that whatever values are assigned to $a$ and $g$, the model assumes a constant decay rate over time for which there is neither a theoretical nor an empirical justification.

Our next criticism of the simple model of equation (10) relates to the fact that such a model (as shown earlier) implies that the process generating mean duration of stay is a deterministic function of time. This assumption cannot be sustained by observations of the real situation; indeed, at best, time can be viewed as a proxy for important determinants of admission policy and length of stay. The length of time that individuals stay in hospital and their probability of admission will be affected by, amongst other factors, the availability of facilities, lengths of waiting lists, current medical practice, patterns of morbidity, and fashion. Moreover, within the linear (or log-linear) static model, the assumption is that each of the observations carries an equal weight. Thus the admission rate (or mean duration of stay) in year $n-10$ is accorded the same importance as in year $n-1$. This is unlikely to be a valid assumption. Last year's practices are much more relevant to this year because the style of medical practice, patterns of morbidity, availability of treatments, etc, will have more in common with those prevailing in the immediate past than those of the more distant past.

Thirdly any forecasting model must be stable over time, otherwise it is of little value. The historical data on mean duration of stay show that it fluctuates from year to year in many specialties. In a static regression model this will be reflected by instability in the value of the coefficients, which will tend to make the forecast unstable. This is shown in fig 3 which gives the 1994 "projection" derived from the 1969-77, 1971-79, 1973-81, and 1977-85 data sets. Alternatively, this instability can be presented in a more compact and elegant way by plotting the coefficients over time (fig 4).

Finally in most cases, although the value of $R^{2}$ obtained is impressive, the Durbin Watson (DW) statistic is very low. A low DW statistic indicates the presence of autocorrelation in the residuals, ie, that there is systematic information that is not accounted for by the regression. Since the usual

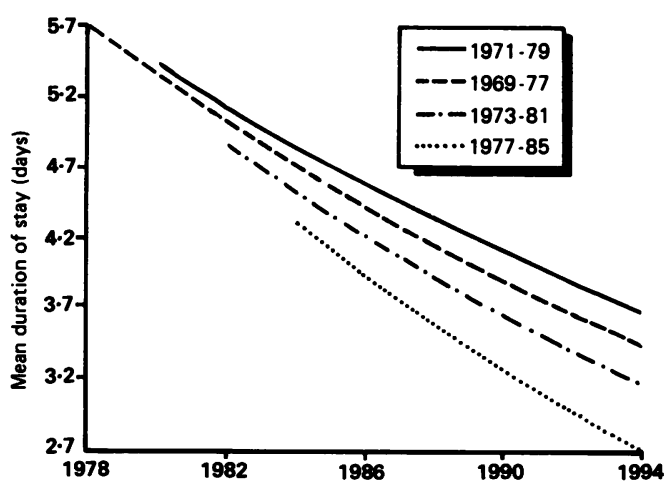

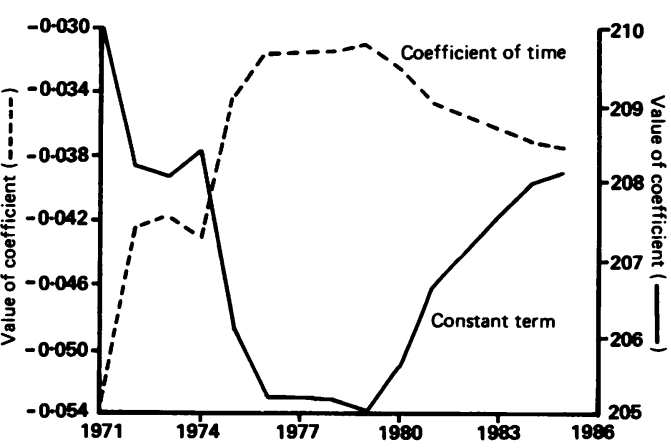

Figure 4 Plot of regression coefficients over time

formula for the variance of the residuals appears in the expression of $R^{2}$, when there is autocorrelation in the residuals this formula overestimates or underestimates the true variance even in large samples, depending on positive or negative correlation of the residuals. Thus $R^{2}$ will overestimate (underestimate) the proportion of the true variance which is taken account of by the linear relationship.

In short the fitted data do not describe the process with an acceptable degree of accuracy. This is not surprising in view of our criticism of the applicability of specification (equation 9) to the current problem and the fact that time is assumed to be the sole determinant of mean duration of stay or admission rate.

To give a concrete example of our suggested method of analysis we applied the Box-Jenkins methodology, outlined above, to the time series of mean duration of stay for general surgery for age group 15-44 (1969-1982). Figure 5 compares the $\log$-linear trend and the Box-Jenkins fit of the data. It is clear from the figure that the time series model mimics the movement of the series,

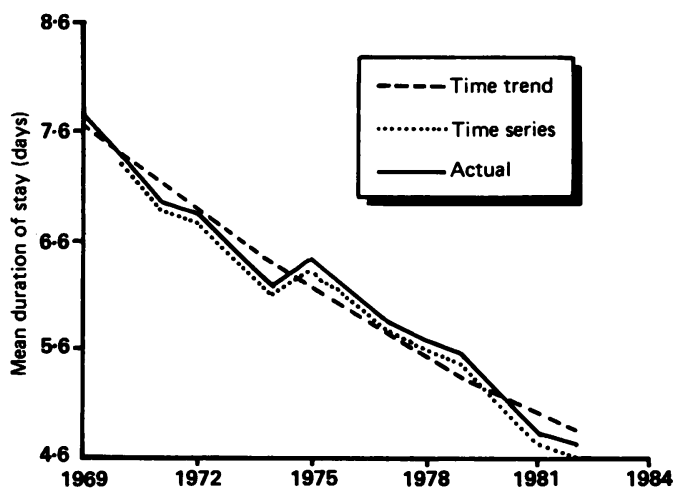

Figure 5 Plot of fitted and actual values for mean duration of stay (days): general surgery (age 15-44 years)

whereas the log-linear model does not. This gain is mainly due to the fact that the information content of the residuals from a log-linear trend has been taken into account in the time series approach.

Relationship between mean duration of stay and admission rate

We have already drawn attention to the fact that, in forecasts of hospital bed requirements derived from equation (1) by incorporating independent 
projections of mean duration of stay, admission rate, and population to be served, the assumption is that variations in each are not correlated. From inspection of the past trends in both mean duration of stay and admission rate, it is clear that changes in some specialty, age, and sex groups tend to correlate. There are empirical reasons to suppose that mean duration of stay and admission rate will not be independently determined. Firstly, there tends to be a finite number of beds and the demand for their use tends to be greater than their supply. Under these circumstances, hospital bed days vacated by a decrease in the average duration of stay are likely to be filled by an increase in the number of admissions to those beds. Secondly, even were there not to be a supply constraint, the policy in some specialties of admitting patients for part of the investigations or treatments they require, discharging them, and then readmitting them for completion of the treatments, will result in a decrease in the mean duration of stay and an increase in admission rate, but will not necessarily effect any change in the gross number of hospital days per patient episode.

If all of the principal components in equation (1) are projected under the assumption that each varies independently of the other, then the likely resuit will be misleading forecasts. Inadequacy of the forecast will be compounded by the fact that none of the parameters is weighted. The reasons for this are as follows. Equation (9) is concerned with rate of change. Consider the situation where, over a long period, both mean duration of stay and admission rate tend to decrease, but in which in the immediate past admission rate increases and there is an increase in the rate of decay of mean duration of stay. The regression will result in a global trend over the whole period and recent changes will tend to be masked. Multiplying the two projections without correction for interdependence will result in either an inflated or deflated value for the bed forecasts.

We conclude that a joint estimation of mean duration of stay and admission rate is likely to result in a more accurate forecast of beds. The approaches outlined above for single equation models can easily be generalised to a multiequation system or vector time series model. Here we attempt to discover the joint distribution of the three important variables involved, ie, the vector [MDS,AR,BEDS]. Such models estimate the following general type of system:

$$
\begin{aligned}
& \mathrm{MDS}=\mathrm{f}\left(\mathrm{BEDS}, \mathrm{AR}, \mathrm{MDS}-{ }_{1}, \mathrm{~W}\right)+\varepsilon_{1} \\
& \mathrm{AR}=\mathrm{f}\left(\mathrm{BEDS}, \mathrm{AR}-{ }_{1}, \mathrm{MDS}, \mathrm{U}\right)+\varepsilon_{2} \\
& \mathrm{BEDS}=(\mathrm{AR} \star \mathrm{MDS} \star \mathrm{POP} \star \varepsilon) / 365
\end{aligned}
$$

where $W$ and $U$ represent one or more exogenous variables and $\varepsilon_{1}, \varepsilon_{2}$ are error terms which are not independent of each other. In this system if $W$ and $U$ were restricted to historical values of the variables involved then the system would be a vector time series model; if in addition they include other exogenous variables, then the system would be a dynamic simultaneous structural model. It is clear that the gain in forecast accuracy will be a consequence of the joint estimation method, and the fact that the disturbance term's correlation structures has been taken into account. The type of data needed to estimate such models is no different to that of the univariate case. Again many of the presently available statistical and managerial packages have facilities to estimate such models.

\section{Conclusion}

There is no doubt that health service planners require access to models for predicting future needs for health care facilities. Forecasting the requirements for acute hospital beds is of particular importance because of the substantial capital and cost implications. Careful examination of the most frequently used method indicates that it does not describe historical patterns of hospital bed utilisation accurately. When it is used to project future requirements, it is inherently unstable. (The reasons for this instability are discussed in detail.) Two alternative approaches to forecasting-time series and structural models-have been discussed. The time series approach seems to offer greater scope for managerial forecasting.

1 Box GEP, Jenkins GM. Time series analysis-forecasting and control. San Francisco: Holden-Day, 1976.

2 Nelson CR. Applied time series analysis for managerial forecasting. San Francisco: Holden-Day, 1973.

3 Granger CWJ Forecasting in business and economics. New York: Academic Press, 1980.

4 Townsend P, Davidson N. Inequalities in health (The Black

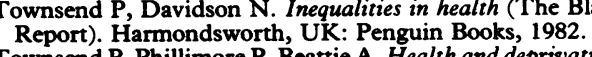

5 Townsend P, Phillimore P, Beattie A. Health and deprivation Inequality in the north. London: Croom Helm, 1988

6 Jarman B. Identification of underprivileged areas. Br Med $\mathcal{F}$ 1983; 286: 1705-8.

7 Department of Health and Social Security. Sharing resources for health in England. Report of the Resource Allocation Working Party (RAWP). London: HMSO, 1976. 